\title{
Herd Immunity: A Realistic Target?
}

\author{
Michael AB Naafs* \\ Dutch Internist-Endocrinologist, Naafs International Health Consultancy, Netherlands
}

Received: 制: September 17, 2018; Published: September 24, 2018

*Corresponding author: Michael AB Naafs, Dutch Internist-Endocrinologist, Health Consultant at Naafs International Health Consultancy, Rhodoslaan 20, 7577KN, Oldenzaal, The Netherlands

\begin{abstract}
In this mini-review herd immunity is discussed by reviewing some recent vaccination campaigns for HPV, MEN-C, MEN-W and Ebola. It is concluded that vaccination coverage is rapidly declining due to societal factors, financial and logistical hurdles. Herd immunity depends now on unpredictable herd effects, Secondary declining transmission dynamics and carriage figures favour strong herd effects. Different target populations in the MEN-W vaccination programs of the UK and the Netherlands don't improve public confidence in an anti-vaxxers climate. Herd immunity is not a realistic target anymore and is dependent on herd effects varying with vaccination coverage percentages and this should be explained to the public in a clear way.
\end{abstract}

\section{Introduction}

The term "herd immunity" was introduced a century ago and used widely after increasing use of vaccines and vaccination protocols in the process of eradication of diseases [1]. The definition of herd immunity varies by several authors. Some use it to describe the proportion immune among individuals in a population, others use it with reference to a particular threshold proportion of immune individuals that should lead to a decline in incidence of infection and still others use it to refer to a pattern of immunity that should protect a population from an invasion of a new infection [2]. A common implication of the term is that the risk of infection among susceptible individuals in a population is reduced by the presence and proximity of immune individuals, this is called the "herd effect" or indirect proportion [2]. Individual immunity is a powerful force affecting host health and pathogen evaluation.

The effects of individual immunity also scale up to affect pathogen transmission dynamics and the success of vaccination campaigns for entire host populations. Population-scale immunity is often termed "herd immunity" [3]. Herd immunity should be in more than $80 \%$ of the population [4]. Apart from innate immunity, it may be acquired either naturally or artificially. If herd immunity is dependent on natural infection, the large proportion of individuals in the population must have been exposed with sufficient frequency to an infectious agent to become immune [5]. It is important to realize that the herd immunity threshold is calculated by mathematical models that can differ for different infectious agents and geographical sites [2]. As vaccines must overcome the challenges of poor immune responses in hosts and antigenic diversity in pathogens, public policy plays a critical role in achieving the high population vaccination rate to achieve herd immunity and freedom of disease [6].

However, for well over a decade multiple studies have demonstrated concerning patterns of decline of confidence in vaccines, manufacturers, the medical professionals who administer vaccines and the scientists who study and develop vaccines [7]. Areas with low vaccination rates have resulted in localized outbreaks of vaccine-preventable diseases, including measles and pertussis [8]. For that reason achieving herd immunity in new vaccination programs has been difficult and the question if reaching herd immunity should be a realistic target is a valid one. To answer that question some recent vaccination campaigns are reviewed here.

\section{HPV Vaccinations}

Modelling studies have been widely used to inform Human Papilloma Virus (HPV) vaccination policy decisions. However, many models exist and it is not known whether they produce consistent predictions of population-level effects and herd effects. Brison et al. searched MEDLINE and EMBASE for transmission-dynamic modelling studies published between Jan 1, 2009 and April 28, 2015 , that predicted the population-level impact of vaccination on HPV 6,11,16 and 18 infections in high -income countries. Strategies investigated were girls-only vaccination and girls and boy's vaccination at 12 years. Base-case vaccine characteristics were $100 \%$ efficacy and lifetime protection. They performed sensitivity analyses by varying vaccination coverage, vaccine efficacy and duration of protection. For all scenarios they pooled model 
predictions of relative reductions in HPV prevalence (RRprev) over time after vaccination and summarized results using the median and 10 th and 90th percentile (80\% uncertainty intervals; UI).

Sixteen of 19 eligible models from ten high -income countries provided predictions. Under base-case assumptions $40 \%$ vaccination coverage and girl only vaccination, the RRprev of HPV 16 among women and men was 0,53 (80\%UI;0,48-0,68) and $0,36(0,28-0,61)$, respectively after 70 years, with $80 \%$ girls only vaccination coverage, the RRprev of HPV 16 among women and men was $0,93(0,90-1,00)$, respectively. Vaccinating boys in addition to girls increased the RRprev of HPV 16 among women and women and men by $0,18(0,13-0,32)$ and $0,35(0,27-0,39)$ for $40 \%$ coverage and $0,07(0,00-0,10)$ and $0,16(0,0-10,25)$ for $80 \%$ coverage, respectively. The RRprev were greater for HPV 6,11 and 18 than for HPV 16 for all scenarios investigated. Finally, at $80 \%$ coverage most models predicted that girls and boy's vaccination would eliminate HPV 6,11,16 and 18 with a median RRprev of 1,00 for women and men for all four HPV types. Variability in pooled findings was low but increased with lower vaccination coverage and shorter vaccine protection (from lifetime to 20 years) [9].

The authors concluded that although HPV models differ in structure, data used for calibration and settings, their populationlevel predictions were generally concordant and suggest that strong herd effects are expected from vaccinating girls only, even with coverage as low as 20\%. Elimination of HPV 16,18,6, and 11 is possible if $80 \%$ coverage in girls and boys is reached and if high vaccine efficacy is maintained over time. HPV vaccination rates are increasing as more children are up to date on HPV vaccination in the U.S. In 2017 roughly, half (49\%) of adolescents were up to date on the HPV vaccine and 66\% of adolescents ages 13-17 years received the first dose to start the vaccine series. On average, the percentage of adolescents who started the HPV vaccine series increased by $5 \%$ each year over the past five years (2013-2017) [10]. However, other countries as e.g. the Netherlands show a sharp drop in HPV vaccination rates.

The Dutch public health institute RIVM has warned that the drop of $15 \% \mathrm{n}$ vaccination of 13 and 14 year old girls over the past two years is extremely worrying and could lead to as many 80 unnecessary additional deaths of cervical cancer each year. The percentage of girls taking up the vaccine fell to a new low of just $45,5 \%$ last year compared to the World Health Organization (WHO) target of 95\%, the level which quarantees "group immunity" from the target disease [11]. The strong herd effects of HPV vaccination are reflected in a recent Cochrane analysis that included 26 trials $(n=73.428)$ [12]. Ten trials with a follow-up of 1,3 to 8 years addressed protection against CIN/AIS (cervical intraepithelial neoplasia, adenocarcinoma in situ). Vaccine safety was evaluated over a period of 6 months to 7 years in 23 studies. Studies were not large enough or of sufficient duration to evaluate cervical cancer outcomes. All but one of the trials was funded by the vaccine manufacturers. Most trials were at low risk of bias. Studies involved monovalent $(\mathrm{n}=1)$, bivalent $(\mathrm{n}=18)$ and quadrivalent vaccines $(n=7)$.
The authors conclude there is a high certainty evidence that HPV vaccines protect against cervical precancer in adolescent girls and young women aged 15-26. The effect is higher for lesions associated with HPV 16,18 than for lesions irrespective of HPV type. The effect is greater for those were negative for hrHPV or HPV 16/18 DNA at enrolment than those unselected for HPV-DNA status. There is moderate certainty evidence that HPV vaccines reduce CIN2+ in older women who are HPV 16/18 negative, but not when they are unselected by HPV-DNA status. No increased risk of serious adverse effects were found. Increased risk of adverse pregnancy outcomes after HPV vaccination cannot be excluded, although the risk of miscarriages and termination are similar between trial arms [12].

\section{MEN Vaccinations}

Recent meningococcal vaccination programs for group C have learned lessons while vaccination programs for meningococcal $\mathrm{W}$ disease have just been started.

MEN-C: Progress $\mathrm{n}$ the prevention and control of meningococcal disease has included the development and introduction of Meningococcal C Conjugate vaccines (MCC). The UK was the first country to implement a national MCC program in 1999, followed by several other European countries, Australia and Canada, all of which have subsequently observed substantial declines in serogroup $C$ disease, which is one of 6 serogroups $(A, B, C, W-135, X$ and $Y$ ) of the 13 recognized serogroups causing meningococcal disease [13-15]. A large part of the success of the MCC vaccine, in common with the glycoconjugate vaccines against Haemophilus influenzae type b (Hib) and major types of Streptococcus pneumoniae are strong herd effects [16]. The epidemiology varies markedly by region $[13,14]$. The most striking contrast is between the "meningitis belt" of sub-Saharan Africa, which is characterized by large, periodic, epidemics of meningitis that occurs during the dry season [17]. In major African epidemics, incidence ranges from 100 to 800 per 100.000 per population, but attack rate may be much higher [17]. In Europe, the Americas and other developed countries disease incidence is typically in the range of 1 and 10 per 100.000 [18].

A number of observations were made with respect to herd immunity and herd effects during MCC introductions. In the Netherlands, a single dose of MCC vaccine at 14 months of age was implemented in the routine program with a one-off-catchup campaign, a decision largely influenced by cost-effectiveness studies [19]. Despite the lack of direct protection in infants, the number of cases in children under 14 months old fell from 20 in 2001 to just 1 in 2004 and the number of cases in adults too old to be targeted also declined [20]. These reductions were most likely due to decreased population transmission following widespread MCC vaccination. The magnitude and duration of these herd effects was largely unexpected ( $>15$ years). In the UK targeting teenagers was crucial in maximizing these indirect effects and to maintain these effects $n$ the longer term [21]. Mathematical models predict these herd effects will persist for several more years, but they do not take in account possible introductions of novel more transmissible serogroup C strains from other countries [21]. 
MEN- W: In February 2015 Public Health England presented data to the Joint Committee on Vaccination and Immunisation (JCVI) on a continuing increase in MEN-W disease in England and Wales since 2009, with the most recent cases in an accelerated manner. JCVI agreed and advised there should be a programme to vaccinate all adolescents 14-18 years. Vaccination of this cohort is expected to protect young people and to provide herd protection for the wider population [22]. By genome library the rise has been identified due to a particular virulent ST-11 strain. This strain is causing severe disease in healthy teenagers and young adults. The strain is more deadly with a higher than usual death rate of $13 \%$. These cases have often different symptoms compared to other kinds of meningococcal diseases. All UK 14-18 years are to be offered MEN- ACWY vaccine by late summer 2017 to prevent further increase in disease among the wider population.

From September 2015 MEN-ACWY vaccine has replaced the MEN-C booster for 14 years old in the UK. Babies in the UK will not be immunized with ACWY, but the MEN-B vaccine, Bexsero, would provide protection against this ST-11 strain [23]. Previous genomic analyses have shown that the ST 11 strain emerged in Brazil in 2003 and then spread to Argentine and Chile. The strain emerged in the UK in 2009 and a further descent of this strain expanded in the UK since 2013. This strain emerged in the Netherlands in 2012 and 2013. Since 2015 the ST 11 strain is also noted in Denmark, France, Spain and Sweden, but at a much lower incidence than in the UK and the Netherlands [24-28]. The annual incidence rate of group W invasive meningococcal disease in the Netherlands increased from $<0,05 / 100.000(\mathrm{n}<10)$ before 2015 to $0,5 / 100.000(\mathrm{n}=80)$ in 2017 . Most isolates (94\%) belong to clonal complex 11 . The incidence rate is highest among $<5$ years old and 15-24 years old. The case fatality rate was $12 \%(17 / 138)$ in 2015-2017. From May 2018, MEN-ACWY vaccination replaces MEN-C vaccination at 14 months and from October 2018 it is planned to offer 13-14 years old MENACWY vaccination.

Teens are now lured to vaccination with a modern real-life victim video campaign as the last HPV vaccination campaign showed historical low coverage of nearly $46 \%$, as mentioned before [11]. This year 130.000 kids are invited and next year 860.000. This is due to limited availability of the vaccine at GSK (Glaxo Smith Kline) and cost-effectiveness reasons [29]. Besides the usual conspiracy theories on social media, new fuss arose when GSK, ironically based on data provided by RIVM, said that the vaccine was ordered too late, despite "GSK advice" Parents of children, who died of meningitis $\mathrm{W}$ accuse Dutch government of negligence now $[30,31]$. Anyway, it is clear that a vaccine coverage of $80 \%$ will not be reached. Both the UK and Dutch RIVM rely on the strong herd effects of the MCC vaccine program in the past, hoping these will be present with ACWY vaccination too.

\section{Ebola Vaccinations}

To illustrate the problems of achieving herd immunity Masterson et al. showed this is not a realistic target for current vaccination strategies, using Ebola outbreaks as an example [32]. They analysed the requirements for a prophylactic program based on the basic reproductive number R0. i.e., the number of secondary cases that result from an individual infection. Published R0 values were determined by systemic literature research and ranged from 0,37 to 20 . R0's $>4$ realistically reflected the critical early outbreak phases and superspreading events. Based on the R0's the herd immunity threshold (Ic) was calculated using the equation Ic=1-(1/ R0). The critical Vaccination Coverage (Vc) needed to provide herd immunity was determined by including the vaccine Effectiveness (E) using the equation Vc=Ic/E. At a R0 of 4, the Ic is $75 \%$ and at an $\mathrm{E}$ of $99 \%$, more than $80 \%$ has to be vaccinated to establish herd immunity.

Such vaccination rates are currently unrealistic because of societal resistance against vaccinations, financial and logistic hurdles and a lack of vaccines that provide long-term protection against all human-pathogenic Ebola viruses. Hence, outbreak management will for the foreseeable future depend on surveillance and case isolation. Clinical vaccine candidates are available for Ebola viruses. Their use will be focused on health-care workers, potentially in combination with ring vaccination approaches. The findings of Masterson et al support the conclusions of the WHO Strategic Advisory Group of Experts (SAGE) meeting n April 2017 [33]. In case of future and recent outbreaks as in DR Congo now, SAGE recommends the use of rVSV-ZEBOV ring vaccination strategies [33,34].

\section{Conclusion}

It is obvious that herd immunity as achieved in former measles and rubella vaccination campaigns (95\% coverage) will not be achieved anymore. The MEN-C vaccination campaign showed that present programs must rely on strong herd effects. The magnitude and duration of these herd effects are unpredictable for future vaccination programs. The last HPV campaign showed a historical low vaccination coverage of nearly $46 \%$ in the Netherlands, but transmission and carriage figures show herd immunity may be sufficient. Conspiracy theories are fed by anti- vaxxers with no accountable leaders, mostly citing a retracted autism study blaming vaccines. However, in the Netherlands, the "Osterhaus Affair" has not raised confidence in vaccination programs, either. In 2009, this Dutch virologist, advised WHO about the handling of an A1H1N! pandemic (Swine Flu, Mexican Flu) that never occurred [35,36]. Twenty of 40 million vaccines doses bought, the Dutch had to throw away.

The different vaccination program approaches of the severe invasive MEN-W serogroup raises new confidence questions, as in the UK all 14- 18 years old are vaccinated while in the Netherlands only 13-14 years old are vaccinated in two tranches for logistical and cost-effectiveness reasons. Waiting for action by "an acceleration of infection numbers" model in the decision making process has received a lot of criticism in mainstream media as acceleration has been observed in the UK already. An Ebola vaccination model applied in DR Congo now shows the limitations of present vaccination programs, Herd immunity is no longer quaranteed with vaccination coverage percentages below $80 \%$. This should be communicated with the public explaining, that herd immunity 
is not a realistic target anymore and relies on unpredictable herd effects varying with different vaccination coverage percentages.

\section{References}

1. Topley WW, Wilson GS (1923) The spread of bacterial infection: the problem of herd immunity. J Hyg 21(3): 243-249.

2. Fine P, Eanes K, Heymann DL (2011) Herd Immunity: A Rough Guide. Clin Infect Dis 52(7): 911-916.

3. Metcalf CJ, Ferrari M, Graham AL, Grenfell BT (2015) Understanding herd immunity. Trends Immunol 36(12): 753-755.

4. Kim TH, Johnston J, Loeb M (2011) Vaccine herd effect. Scand J Inf Dis 43: 683-689.

5. Weiler JL (2017) Natural vs. artificial herd immunity: What have failed vaccination programs taught us? Children's Health Défense.

6. Mallory ML, Lindesmith LC, Baric RS (2018) Vaccination-induced herd immunity: Successes and challenges. J Allergy Clin Immunol 142(1): 6466.

7. Smith TC (2017) Vaccine rejection and hesitancy: A review and call to action. Open Forum Inf Dis 4(3): 146.

8. Phadke VK, Bednarczyk RA, Salmon DA, Omer SB (2016) Association between vaccine refusal and vaccine- preventable disease in the united states: A review of measles and pertussis. JAMA 315(11): 1149-1158.

9. Brisson M, Bénard É, Drolet M, Bogaards JA, Baussano I, et al. (2016) Population-level impact, herd immunity and elimination after human papillomavirus vaccination: A systematic review and metaanalysis of predictions from transmission-dynamics models. Lancet Public Health 1(1): e8-e17.

10. Human Papillomavirus (HPV) (2018) Vaccination Coverage Data.

11. Cluskey P (2018) Sharp drop in number of Dutch teenage girls getting HPV vaccination. The Irish Times 18: 22

12. Arbyn M, Xu L, Simoens C, Martin-Hirsch PP (2018) Prophylactic vaccination against human papillomaviruses to protect cervical cancer and its precursors. Cochrane Database Syst Rev Vol. 5.

13. Stephens DS, Greenwood B, Brandtzaeg P (2007) Epidemic meningitis, meningococcaemia and Neisseria meningitides. Lancet 369(9580): 2196-2210.

14. Girard MP, Preziosi MP, Aguado MT, Kieny MP (2006) A review of vaccine research and development: Meningococcal disease. Vaccine 24(22): 4692-7000.

15. Trotter CL, Ramsey ME (2007) Vaccination against meningococcal disease in Europe: Review and recommendations for the use of conjugate vaccines. FEMS Microbiol Rev 31: 101-107.

16. Trotter CL, McVernon J, Ramsay ME, Whitney CG, Mulholland EK, et al. (2008) Optimising the use of conjugate vaccines to prevent disease caused by Haemophilus influenzae type b, Neisseria meningitides and Streptococcus pneumoniae. Vaccine 26(35): 4434-4445.

17. Greenwood B (1999) Manson Lecture: Meningococcal meningitis in Africa. Trans R Soc Trop Med Hyg 93(4): 341-353.

18. Lystad A, Aasen S (1991) The epidemiology of meningococcal disease in Norway 1975-91. NLPH Ann 14(2): 57-65.

19. Welte R, Van den Dobbelsteen G, Bos JM, de Melker H, Van Alphen L, et al. (2004) Economic evaluation of meningococcal serogroup $C$ conjugate vaccination programmes in the Netherlands and its impact on decision making. Vaccine 23(4): 470-479.

20. De Greeff SC, de Melker HE, Spanjaard L, Schouls LM, van Derende A (2006) Protection from routine vaccination at the age of 14 months with meningococcal serogroup $C$ conjugate vaccine in the Netherlands. Paediatr Infect Dis J 25(1): 79-80.

21. Balmer P, Burman C, Serra L, York LJ (2018) Impact of meningococcal vaccination on carriage and disease transmission: A review of the literature. Hum Vacc Immnother 14(5): 1118-1130.

22. NHS (2018) Meningococcal ACWY (MEN ACWY) vaccination programme 2018/19. https://wwwdigital.nhs.uk/services/generalpractice-collections/meningo coccal-acwy-menacwy-vaccinationprogramme-2018/19/

23. Meningococcal Group W (2015) A new virulent strain of meningitis and septicaemia. https://www.meningitis.org/meningitis/what-ismeningitis/bacterialmeningitis/meningococcal-group-W(menW/

24. Lucidarme J, Hill DM, Bratcher HB, Gray SJ, du Plessis M, et al. (2015) Genomic resolution of an aggressive, widespread, diverse and expanding meningococcal serogroup B, C and W lineage. J Infect 7(5): 544-552.

25. Ladhani SN, Beebeejaun K, Lucidarme J, Campbell H, Gray S, et al. (2015) Increase in endemic Neisseria meningitides capsular group W sequence type 11 complex associated with severe invasive disease in England and Wales. Clin Inf Dis 60(4): 578-585.

26. Lucidarme J, Scott KJ, Ure R, Smith A, Lindsay D, et al. (2015) An international invasive meningococcal disease outbreak due to a novel and rapidly expanding serogroup W strain, Scotland and Sweden, July to August 2015. Euro Surveill 21(45): 30395.

27. Knol MJ, Hahné SJM, Lucidarme J, Campbell H, de Melker HE, et al. (2017) Temporal association between national outbreaks of meningococcal serogroup $\mathrm{W}$ and $\mathrm{C}$ disease in the Netherlands and England: An observational cohort study. Lancet Public Health 2(10): e473-e482.

28. Knol MJ, Ruijs WL, Antonise Kamp L, de Melker HE, van der Ende A Implementation of MEN ACWY vaccination because of ongoing increase in serogroup W invasive meningococcal disease, the Netherlands, 2018. Euro Surveill 23(16) 2018;sept. 10.

29. Keulemans M (2018) Campagne met patienten moet tiener aan de meningokokken prik krijgen. de Volkskrant.

30. Efting M, Miserus C Overheid wist in 2016 al van mogelijke meningokokken uitbraak. de Volkskrant, 2018; sept 14.

31. Efting M., Miserus M (2018) Als de overheid eerder had beslist,had mijn zoon nog geleefd. de Volkskrant, 2018; sept 14.

32. Masterson SG, Lobel L, Carroll MW, Wass MN, Michaelis M (2018) Herd Immunity to Ebolaviruses Is Not a Realistic Target for Current Vaccination. Front Immunol 9: 1025.

33. WHO (2017) Meeting of the Strategic Advisory Group of Experts on Immunization. Wkly Epidemiol Rec 92: 301-320.

34. Schnirring L (2018) Ebola infects 5 more in DRC, lifting total to 137. CIDRAP News, 2018; sep 13.

35. Cohen D, Carter P (2010) WHO and the pandemic flu "conspiracies". 340: c2912.

36. Enserink M Swine flu names evolving faster than swine flu itself. Science. 2019; May 8 and https://www.sciencmag.org/news/200/05/swineflu/ 


\section{ISSN: 2574-1241}

DOI: 10.26717/BJSTR.2018.09.001779

Michael AB Naafs. Biomed J Sci \& Tech Res

(c) (i) This work is licensed under Creative

Submission Link: https://biomedres.us/submit-manuscript.php

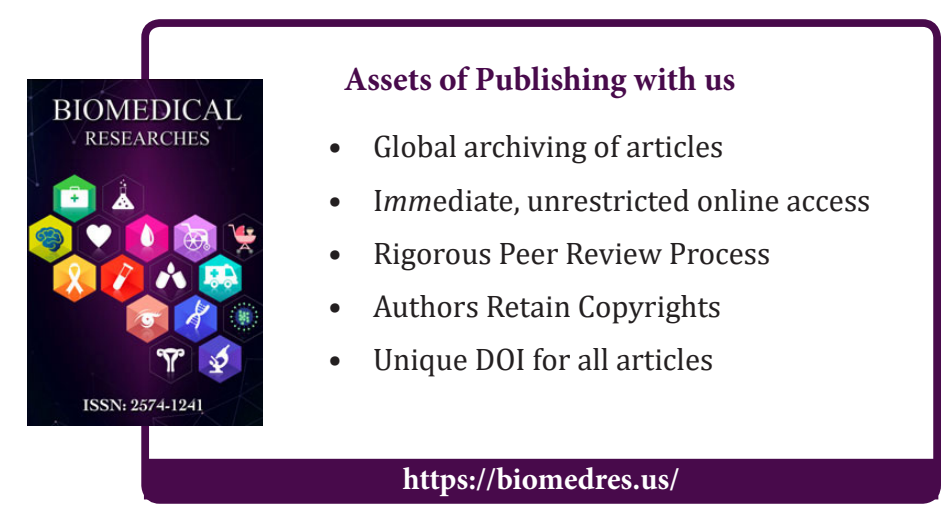

